\title{
PURE STATES ON FREE GROUP C*-ALGEBRAS
}

\author{
CHARLES AKEMANN \\ Department of Mathematics, University of California, Santa Barbara, CA 93106, USA \\ e-mail: akemann@math.ucsb.edu \\ SIMON WASSERMANN \\ Department of Mathematics, University of Glasgow, Glasgow G12 8QW, UK \\ e-mail:asw@maths.gla.ac.uk \\ and NIK WEAVER \\ Department of Mathematics, Washington University in Saint Louis, Saint Louis, MO 63130, USA \\ e-mail: nweaver@math.wustl.edu
}

(Received 1 June 2007; accepted 10 November 2007)

\begin{abstract}
We prove that all the pure states of the reduced $\mathrm{C}^{*}$-algebra of a free group on an uncountable set of generators are *-automorphism equivalent and extract some consequences of this fact.
\end{abstract}

2000 Mathematics Subject Classification. 46L05.

1. Preliminaries. For any set $R$ with two or more elements, let $F_{R}$ denote the free group on $R$ with generators $\left\{u_{r}: r \in R\right\}$ and let $C_{r}^{*}\left(F_{R}\right)$ denote the reduced group $\mathrm{C}^{*}$ algebra. We shall not distinguish between the elements of $F_{R}$ and the corresponding unitary operators in $C_{r}^{*}\left(F_{R}\right)$. In what follows, $r_{0}$ will be a fixed element of $R, u$ will denote the generator $u_{r_{0}}$ and $F_{u}$ will denote the subgroup of $F_{R}$ generated by $u$. We view $C_{r}^{*}\left(F_{S}\right)$ as the $C^{*}$-subalgebra of $C_{r}^{*}\left(F_{R}\right)$ generated by the unitaries $\left\{u_{s}: s \in S\right\}$ and $C_{r}^{*}\left(F_{u}\right)$ as the $C^{*}$-subalgebra generated by $u$. Let $P_{u}$ (resp. $P_{S}$ ) denote the unique trace preserving conditional expectation from $C_{r}^{*}\left(F_{R}\right)$ onto $C_{r}^{*}\left(F_{u}\right)$ (respectively $C_{r}^{*}\left(F_{S}\right)$ ). Recall that $C_{r}^{*}\left(F_{u}\right)$ is *-isomorphic to the *-algebra of continuous complex valued functions on the unit circle, with $u$ going into the function $\theta(z)=z$. Let $f_{0}$ denote the (unique!) pure state of $C_{r}^{*}\left(F_{u}\right)$ that satisfies $f_{0}(u)=1$ and let $f=f_{0} \circ P_{u}$.

2. Results. If $R$ is uncountable, then $C_{r}^{*}\left(F_{R}\right)$ is inseparable. For $\operatorname{Card}(R)=\aleph_{1}$, the algebra $C_{r}^{*}\left(F_{R}\right)$ is discussed in [11, Corollary 6.7], where it is shown that $C_{r}^{*}\left(F_{R}\right)$ is inseparable, but that every abelian subalgebra is separable. Powers [12] showed that for $\operatorname{Card}(R)=2, C_{r}^{*}\left(F_{R}\right)$ is simple and has unique trace. Powers' method extends to general $R$. For general free products of groups, simplicity and uniqueness of trace follow by results of Avitzour [7]. In [1] and [3] the methods of [4] were used to extend the simplicity and uniqueness of trace results to a host of other group of $\mathrm{C}^{*}$-algebras where free sets lurked in the underlying groups. In [6] Archbold also obtained related results.

Lemma 2.1. If $S \subset R$, Card $(S)>1$ and $\alpha$ is a ${ }^{*}$-automorphism of $C_{r}^{*}\left(F_{S}\right)$, then $\alpha$ has an extension to $a^{*}$-automorphism of $C_{r}^{*}\left(F_{R}\right)$.

Proof. Check that if $\alpha^{\prime}$ is defined on the *-algebra $A$ generated by $C_{r}^{*}\left(F_{S}\right)$ and the generators in $R \backslash S$ by applying $\alpha$ to elements of $C_{r}^{*}\left(F_{S}\right)$ and leaving the other generators 
alone, then $\alpha^{\prime}$ is a *automorphism of $A$. Every element of $C_{r}^{*}\left(F_{R}\right)$ is representable in the form of an element of $l^{2}\left(F_{R}\right)$, and the trace of such an element is simply the coefficient of the identity. Since the trace is unique on $C_{r}^{*}\left(F_{S}\right)$ by [1, Proposition 1] (see also [7, 3.1]), $\alpha$ preserves the trace. Thus it is easy to verify that $\alpha^{\prime}$ preserves the trace on $A$. Again by density of $A$ in $l^{2}\left(F_{R}\right)$, for any $a \in A$ and any $\epsilon>0$ there exists $b \in A$ such that $\|b\|_{2}=1$ and $\|a b\|_{2}>\|a\|-\epsilon$. So $\left\|\alpha^{\prime}(b)\right\|_{2}=\|b\|_{2}=1$ by invariance of the trace, and hence $\left\|\alpha^{\prime}(a)\right\| \geq\left\|\alpha^{\prime}(a) \alpha^{\prime}(b)\right\|_{2}=\left\|\alpha^{\prime}(a b)\right\|_{2}=\|a b\|_{2}>\|a\|-\epsilon$. Since a similar inequality holds for $\alpha^{-1}$, we see that $\alpha^{\prime}$ extend by continuity to an automorphism of $C_{r}^{*}\left(F_{R}\right)$.

LEMMA 2.2. The state $f$ is the unique state extension of $f_{0}$ to $C_{r}^{*}\left(F_{R}\right)$, and $f$ is a pure state of $C_{r}^{*}\left(F_{R}\right)$. Moreover, $\left.f\right|_{C_{r}^{*}\left(F_{\mathrm{S}}\right)}$ is pure for any subset $S$ of $R$ that contains $r_{0}$.

Proof. Let $g$ be a state of $C_{r}^{*}\left(F_{R}\right)$ such that $g(u)=1$. The Cauchy-Schwarz inequality applies to show that $g((1-u) a)=g(a(1-u))=0$ for any $a \in C_{r}^{*}\left(F_{R}\right)$. By induction, $g\left(u^{n}\right)=g\left(u^{-n}\right)=1$ for every natural number $n$. Fix $s \in F_{R} \backslash F_{u}$. By the Cauchy-Schwarz inequality again, as above, $g\left(u^{n} s u^{-n}\right)=g(s)$ for every natural number $n$. Taking $\xi$ to be the canonical trace vector in $l^{2}\left(F_{R}\right), l^{2}\left(F_{R}\right)=H_{0} \oplus H_{1}$, where $H_{0}$ is the closed linear span of all vectors of form $w \xi$ with $w$ a reduced word in $F_{R}$ with a non-zero power of $u$ on the left, and $H_{1}$ is the closed linear span of those $w \xi$ with $w$ not ending in a non-zero power of $u$ on the left. Then, $u^{n} H_{1} \subset H_{0}$ for any non-zero integer $n$ and $s H_{0} \subset H_{1}$. By [8, Lemma 2.2] (see also [7, Lemma 3.0])

$$
|g(s)|=\lim _{k \rightarrow \infty}\left|(1 / k) \sum_{n=1}^{k} g\left(u^{n} s u^{-n}\right)\right| \leq \lim _{k \rightarrow \infty}\left\|(1 / k) \sum_{n=1}^{k} u^{n} s u^{-n}\right\| \leq \lim _{k \rightarrow \infty} \frac{2}{\sqrt{k}}=0 .
$$

By linearity and continuity of $g$, this implies that $g=\left.g\right|_{C_{r}^{*}\left(F_{u}\right)} \circ P_{u}$ and hence that $g=f$. An easy convexity argument shows that $f$ is a pure state.

The conclusion of the last sentence of the Lemma follows immediately from the conclusion of the first sentence.

PROPOSITION 2.3. Let $\left\{G_{r}\right\}_{r \in R}$ be a set of nontrivial countable groups and for nonempty $S \subset R$, let $G_{S}$ be the free product $\left(*_{r \in S} G_{r}\right)$. Given a nonempty countable subset $S_{0}$ of $R$, if $g$ is a pure state on $C_{r}^{*}\left(G_{R}\right)$ there is a countable subset $S$ of $R$ containing $S_{0}$ such that $\left.g\right|_{C_{r}^{*}\left(G_{S}\right)}$ is a pure state of $C_{r}^{*}\left(G_{S}\right)$. Moreover, $C_{r}^{*}\left(G_{S}\right)$ is separable and also simple if $\left|G_{s}\right|>2$ for some $s \in S$.

Proof. Assume without loss of generality that $R$ is uncountable. For any nonempty countable $S \subset R, C_{r}^{*}\left(G_{S}\right)$ is separable, and by [7,3.1] simple if $\left|G_{s}\right|>2$ for some $s \in S$. If $\left(\pi_{g}, H_{g}, \xi_{g}\right)$ is the representation of $C_{r}^{*}\left(G_{R}\right)$ corresponding to $g$ by the Gelfand-Naimark-Segal construction, sequences of sets

$$
S_{1} \subset S_{2} \subset \cdots \subset R
$$

with each $S_{i}$ countably infinite, closed separable linear subspaces

$$
\mathbb{C} \xi_{g}=H_{1} \subset H_{2} \subset \cdots \subset H_{g}
$$

and, for each $i \geq 2$, a countable dense subset $X_{i}$ of the unit sphere of $H_{i}$ such that

$$
X_{2} \subset X_{3} \subset \cdots
$$


are constructed inductively so that

$$
\pi_{g}\left(C_{r}^{*}\left(F_{S_{i}}\right)\right) H_{i} \subseteq H_{i+1}
$$

for $i \geq 1$. Let $S_{1}$ be a non-empty countable subset of $R$ containing $S_{0}$. For the inductive step, given $S_{i}$ and $H_{i}$, let $H_{i+1}$ be the closed linear span of $\pi_{g}\left(C_{r}^{*}\left(G_{S_{i}}\right)\right) H_{i}$, which is separable, and let $X_{i+1}$ be a countable dense subset of the unit sphere of $H_{i+1}$ containing $X_{i}$. By Kadison's transitivity theorem there is a countable set $\mathcal{U}_{i+1}$ of unitaries in $C_{r}^{*}\left(G_{R}\right)$ such that for $\xi, \eta \in X_{i+1}, \pi_{g}(u) \xi=\eta$ for some $u \in \mathcal{U}_{i+1}$. Since each such $u$ is a normlimit of a sequence of finite linear combinations of elements of $G_{R}$, there is a countable subset $S_{i+1}^{\prime}$ of $R$ such that $\mathcal{U}_{i+1} \subset C_{r}^{*}\left(G_{S_{i+1}^{\prime}}\right)$. Let $S_{i+1}=S_{i+1}^{\prime} \cup S_{i}$. Now let

$$
S=\bigcup_{i=1}^{\infty} S_{i}, \quad X=\bigcup_{i=2}^{\infty} X_{i}, \quad H=\overline{\bigcup_{i=1}^{\infty} H_{i}}
$$

Then $S$ and $X$ are countable, $H$ is separable, $\pi_{g}\left(C_{r}^{*}\left(G_{S}\right)\right) H \subseteq H$ and $X$ is dense in the unit sphere of $H$. If $\xi, \eta \in X$, then $\pi_{g}(v) \xi=\eta$ for some unitary $v \in C_{r}^{*}\left(G_{S}\right)$. It follows that for any $\varepsilon>0$ and unit vectors $\xi, \eta \in H,\left\|\pi_{g}(w) \xi-\eta\right\|<\varepsilon$ for some unitary $w \in C_{r}^{*}\left(G_{S}\right)$, which implies that $\left.\pi_{g}\left(C_{r}^{*}\left(G_{S}\right)\right)\right|_{H}$ acts irreducibly on $H$. Since $\left.g\right|_{C_{r}^{*}\left(G_{S}\right)}$ is the state of $C_{r}^{*}\left(G_{S}\right)$ corresponding to $\xi_{g},\left.g\right|_{C_{r}^{*}\left(G_{S}\right)}$ is pure.

THEOREM 2.4. Any two pure states of $C_{r}^{*}\left(F_{R}\right)$ are *-automorphism equivalent.

Proof. If $R$ is countable, the conclusion is immediate from [10]. Assume that $R$ is uncountable. Let $g$ be a pure state of $C_{r}^{*}\left(F_{R}\right)$. We shall show that $g$ is *automorphism equivalent to $f$. By Proposition 2.3 there is a countably infinite subset $S \subset R$ such that $r_{0} \in S$ and $\left.g\right|_{C_{r}^{*}\left(F_{\mathrm{S}}\right)}$ is pure. We have already noted that $C_{r}^{*}\left(F_{S}\right)$ is simple, and it is obviously separable, so by [10] choose a *automorphism $\gamma_{0}$ of $C_{r}^{*}\left(F_{S}\right)$ such that $\left.g\right|_{C_{r}^{*}\left(F_{S}\right)}=\gamma_{0}^{*}\left(\left.f\right|_{C_{r}^{*}\left(F_{S}\right)}\right)$. By Lemma 2.1, extend $\gamma_{0}$ to a ${ }^{*}$-automorphism $\gamma$ of $C_{r}^{*}\left(F_{R}\right)$. We must show that $\gamma^{*}(f)=g$. Lemma 2.2 shows that $\left.f\right|_{C_{r}^{*}\left(F_{S}\right)}$ has unique state extension to $C_{r}^{*}\left(F_{R}\right)$. Since $\gamma$ is a *automorphism extending $\gamma_{0}$, the same uniqueness of state extension must follow for $\gamma^{*}\left(\left.f\right|_{C_{r}^{*}\left(F_{S}\right)}\right)=\left.g\right|_{C_{r}^{*}\left(F_{S}\right)}$. Thus $\gamma^{*}(f)=g$.

The next result is in contrast to Corollary 0.9 of [5].

THEOREM 2.5. If $g$ is a pure state on $C_{r}^{*}\left(F_{R}\right)$, then its hereditary kernel,

$$
\left\{a \in C_{r}^{*}\left(F_{R}\right): g\left(a^{*} a+a a^{*}\right)=0\right\},
$$

contains a sequential abelian approximate unit, and hence a strictly positive element.

Proof. By Theorem 2.4 it suffices to prove this for $f$. Choose an excising sequence $\left\{a_{n}\right\}$ for $f_{0}$ in $C_{r}^{*}\left(F_{u}\right)$, as defined in [2]. Let $p=\lim a_{n}$ in $C_{r}^{*}\left(F_{R}\right)^{* *}$. By Lemma 2.2, $p$ is a minimal projection there. By [2, Prop. 2.2], $\left\{a_{n}\right\}$ will excise $f$ and $\left\{1-a_{n}\right\}$ will be an approximate unit for $\left\{a \in C_{r}^{*}\left(F_{R}\right): f\left(a^{*} a+a a^{*}\right)=0\right\}$, so $\sum_{i}^{\infty} 2^{-n}\left(1-a_{n}\right)$ is strictly positive there.

THEOREM 2.6. Let $r_{0} \in S \subset R$.

1. Any pure state of $C_{r}^{*}\left(F_{S}\right)$ has a unique extension to a pure state of $C_{r}^{*}\left(F_{R}\right)$.

2. The projection $P_{S}$ is the unique conditional expectation of $C_{r}^{*}\left(F_{R}\right)$ onto $C_{r}^{*}\left(F_{S}\right)$. 
Proof. 1. By Theorem 2.4, any pure state of $C_{r}^{*}\left(F_{S}\right)$ is *-automorphism equivalent to $\left.f\right|_{C_{r}^{*}\left(F_{S}\right)}$, and thus has the unique extension property since Lemma 2.2 shows that $\left.f\right|_{C_{r}^{*}\left(F_{S}\right)}$ has that property.

2. If there were another conditional expectation $Q: C_{r}^{*}\left(F_{R}\right) \rightarrow C_{r}^{*}\left(F_{S}\right)$ distinct from $P_{S}$, then the duals $Q^{*}$ and $P_{S}^{*}$ would have to be different on some element of $C_{r}^{*}\left(F_{S}\right)^{*}$, hence on some state of $C_{r}^{*}\left(F_{S}\right)$, hence on some pure state of $C_{r}^{*}\left(F_{S}\right)$ by the Krein Milman Theorem [9, p. 32]. This is impossible by part 1 of this theorem.

3. Concluding remarks. 1. A very similar proof to that of Proposition 2.3 shows the related result that if $B$ is a separable $C^{*}$-subalgebra of an inseparable $C^{*}$-algebra $A$, then if $g$ is a pure state of $A$, there is a separable $\mathrm{C}^{*}$-subalgebra $C$ of $A$ such that $B \subseteq C$ and $\left.g\right|_{C}$ is pure. An analogous induction argument shows moreover that if $A$ is simple, then a simple $C$ with these properties can be found.

2. The proof of Theorem 2.4 and the preceding lemmas generalize in an obvious way to the general free product groups $G_{R}=*_{r \in R} G_{r}$ considered in Proposition 2.3, provided that one of the constituent groups $G_{r_{0}}$ is abelian with an element of infinite order. Thus any two pure states of $C_{r}^{*}\left(G_{R}\right)$ are *-automorphism equivalent. The corresponding generalizations of Theorems 2.5 and 2.6 to these free product groups then follow, with $G_{r_{0}}$ taking the place of $F_{u}$.

\section{REFERENCES}

1. C. A. Akemann, Operator algebras associated with Fuchsian groups, Houston J. Math. 7(3) (1981), 295-301.

2. C. A. Akemann, J. Anderson and G. K. Pedersen, Excising states of $\mathrm{C}^{*}$-algebras, Canad. J. Math. 38(5) (1986), 223-230.

3. C. A. Akemann and T.-Y. Lee, Computing norms in group $\mathrm{C}^{*}$-algebras, Indiana $U$. Math. J. 29(4) (1980), 505-511.

4. C. A. Akemann and P. A. Ostrand, Simple $C^{*}$-algebras associated with free groups, Amer. J. Math. 98(4) (1976), 1015-1047.

5. C. A. Akemann and N. Weaver, Classically normal pure states, Positivity.

6. R. Archbold, A mean ergodic theorem associated with the free group on two generators, J. Lond. Math. Soc. 13(2) (1976), 339-345.

7. D. Avitsour, Free products of C*-algebras, Trans. A.M.S. 271 (1982), 423-435.

8. M.-D. Choi, A simple $C^{*}$-algebra generated by two finite-order unitaries, Canad. J. Math. 21 (1979), 867-880.

9. R. V. Kadison and J. Ringrose, Fundamentals of the theory of operator algebras, vol. 1 (Academic Press, London, 1983).

10. A. Kishimoto, N. Ozawa and S. Sakai, Homogeneity of the pure state space of a separable C*-algebra, Canad. Math. Bull. 46 (2003), 365-372.

11. S. Popa, Orthogonal pairs of *-subalgebras in finite von Neumann algebras, J. Operator Theory 9 (183), 253-268.

12. R. Powers, Simplicity of the $C^{*}$-algebra associated with the free group on two generators, Duke Math. J. 42 (1975), 151-156. 